\title{
La dimensión científica del psicoanálisis
}

RESUMEN: Se revisa la relación entre la neurociencia y el psicoanálisis. La neurociencia puede proporcionar una nueva base para el crecimiento futuro del psicoanálisis sin que ello suponga un abandono del conjunto enorme de conocimientos clínicos valiosos que el psicoanálisis ha reunido durante el último siglo.

PALABRAS CLAVE: psicoanálisis, neurociencia, multideterminismo, Edipo.
SUMMARY: The relationship between neuroscience and psychoanalysis are examined. Neuroscience could provide a new foundation for the future growth of psychoanalysis an this statement no need lead to abandonment of the vast array of valid clinical knowledge psychoanalysis has accumulated in the course of the past century

KEY WORDS: psychoanalysis, neuroscience, multideterminism, Edipo.

\section{Introducción}

He leído con interés el trabajo "Consciencia, Cientificismo y Multideterminismo en Psicoanálisis" firmado por C. Paniagua (1). La relación del psicoanálisis con las ciencias empíricas denominadas afines no es nada fácil en la actualidad. Por ello se lee con agrado un artículo sobre psicoanálisis conceptualmente preciso, bien documentado, pero que no resulta hermético. El autor defiende que las funciones psíquicas son tan directamente observables como la actividad sináptica y recuerda la multideterminación que preside la acción humana. Critica que la única función legítima de la investigación psiquiátrica sea "ir en pos de los principios biológicos o, como mucho, reflexológicos que subyacen a las manifestaciones psicológicas complejas, suponiendo que solamente por medio del conocimiento de las leyes fisiologicas puede verdaderamente llegarse a conocer conductas que implican intencionalidad, significado y finalidad", pues "la lectura de las intenciones o coherencias en las acciones y experiencias de otros no puede hacerse en los acontecimientos fisiológicos, al menos no más de lo que la finalidad del transporte puede ser colegida a partir del funcionamiento de la mecánica del automóvil". En la misma línea, reclama un espacio para investigar la experiencia subjetiva humana e incide en una cuestión fundamental: el objeto de estudio del psicoanálisis. Este no es sino "el material verbal del paciente que expresa su versión subjetiva del mundo", ya que, "lo que se analiza no es la vida del paciente, sino su psiquismo".

Efectivamente, el estudio del psiquismo, consciente e inconsciente, es la tarea que mejor distingue el psicoanálisis del resto de terapias. Pero, ¿con qué modelo? La historia 
del psicoanálisis ha sido testigo del nacimiento de distintas escuelas psicoanalíticas, muchas de las cuales han propuesto modelos diferentes para la compresión del psiquismo inconsciente. No hay duda que el modelo propuesto por Freud sigue siendo el más completo y elaborado. Sin embargo, conviene recordar que el modelo freudiano estuvo desde el principio y, a lo largo de su extenso desarrollo, apoyado en los conocimientos científicos de la época. La obra de Preyer, Baldwin, Romanes o Darwin subyace en las hipótesis psicoevolutivas de Freud, y los textos de Herbart, Fechner, Meynert o Wernicke influyeron en sus concepciones psicofísicas (2). Por ello, es legítima la pregunta, ¿cuál sería la actitud de Freud frente a la extensa y rica producción científica contemporánea? En el siglo XXI, ¿habría introducido modificaciones en su modelo de aparato psíquico? Diversos autores (3) (4) están convencidos que, como hizo a lo largo de su dilatada existencia, Freud no hubiera permanecido ciego frente a los descubrimientos científicos actuales.

Es posible, que la mayoría de las intervenciones realizadas en el campo psicoanalítico no sean susceptibles de evaluación empírica. Sin embargo, la clínica se sustenta en un edificio teórico muy complejo y tras las teorías subyace el conocimiento científico de cada época -además de las influencias sobre Freud, podríamos mencionar la relación de las teorías de Bion con el modelo matemático de Poincare. Es en este terreno donde las hipótesis psicodinámicas podrían y deberían testarse. Por tanto, el objetivo de este artículo será doble. En primer lugar, se pondrá un ejemplo ilustrativo de cómo la neurociencia actual podría facilitar información que corroborase algunas hipótesis psicoanalíticas clásicas. Para ello se comentarán diversas investigaciones sobre el funcionamiento del cerebelo. En segundo lugar, se planteará el posible papel de la neurociencia como instrumento clarificador de las serias divergencias teóricas que han dividido y continúan oponiendo a los psicoanalistas. Desgraciadamente, la mayoría de estas discrepancias se han saldado en escisiones y disputas personales entre los miembros de los numerosos institutos psicoanalíticos hasta el punto que, los psicoanalistas, constituyen uno de los colectivos con peores relaciones profesionales. En mi opinión, algunas de estas disensiones son susceptibles de dirimirse en su origen: el conocimiento científico que sustenta en parte las teorías en competencia y que permanece oculto al socaire del fragor de la disputa. Con este fin se presentarán algunos hallazgos sobre la maduración cerebral que acompaña a la mielinización interhemisférica.

\section{El cerebelo y las experiencias infantiles tempranas}

Una de las hipótesis psicodinámicas fundamentales sostiene que las experiencias infantiles tempranas condicionan de manera notable el desarrollo y funcionamiento de aparato psíquico adulto. Pues bien, la investigación con técnicas de imagen cerebral ha mostrado al cerebelo, un órgano fundamental en la integración visomotora temprana (5), muy activo desde el nacimiento y a lo largo de todo el primer año de vida. El cerebelo constituye el substrato del sistema mnémico más primitivo, el cual conserva y organiza las memorias más arcaicas, especialmente las relativas a la experiencia motora, pero 
también las originadas a partir de otras modalidades sensoriales (6). Con las primeras experiencias el cerebelo crea mapas o planos del propio self y del mundo circundante que permiten desarrollar un modelo de self-en-el-mundo (7). En este proceso influyen factores constitucionales -en niños autistas se han descrito cambios del funcionamiento cerebeloso- y ambientales que van más allá de la mera estimulación precoz [-se recomienda la lectura de los trabajos de Stern donde desarrolla los conceptos de percepción amodal y entonamiento afectivo (8)]. Estos hallazgos son apasionantes, pero el dato que interesaría al investigador psicoanalítico sería aquel que permitiera la transformación de un conocimiento de orden biológico en conocimiento de orden psicológico, ergo, susceptible de enriquecer la metapsicología. Por este motivo es trascendente lo que sucede cuando en el curso del primer año el cerebelo conecta con el tálamo y el cortex parietal. Mientras estas estructuras van madurando, activan sus propios sistemas mnémicos y son capaces de crear sus propios mapas de la experiencia, los mapas cerebelosos previos no se destruyen. No sólo no se destruyen sino que la información procedente de los mapas cartografiados por el cerebelo perdura, se comparte e influencia la elaboración de los nuevos mapas/representaciones tálamocorticales. Es decir, las memorias más arcaicas van a ser transferidas a los centros superiores y casi nada de nuestra biografía se va a perder. Por tanto, el nuevo modelo de self-en-el-mundo, llamémoslo "cortico-límbico", que albergará representaciones complejas del self, del mundo y de las relaciones entre ambos, no se va a crear desde la nada sino bajo la influencia de la experiencia previa cerebelosa. De hecho, el cerebelo sigue ejerciendo cierto control sobre las funciones cognitivas del adulto y, por eso, ya no se considera un mero órgano de control del movimiento (9). Pero, aún hay más, no es sólo el "contenido" de la experiencia primitiva el que modela y da significado a la adquisición de información nueva, sino que con el "continente" sucede lo mismo. El método con que se elaboran mapas, se procesa la información y se crean modelos internos de organización de la experiencia sensorial también va a perdurar. Este funcionamiento tiene su lógica pues, en cualquier proceso computacional, para que la mayoría de los programas puedan funcionar correctamente son necesarios ciertos programas básicos. En este sentido, los sistemas mnémicos cerebelosos son los mapas del SNC necesarios para poder "mapear" $(7,9)$. Estas hipótesis, aunque basadas en hallazgos empíricos, son todavía especulativas, pero son una muestra del tipo de relación que puede establecerse entre la neurociencia y el psicoanálisis durante el recién comenzado siglo XXI.

\section{Mielinización interhemisférica y complejo de Edipo.}

En palabras del propio Freud "si el psicoanálisis no tuviese otro mérito que la revelación del complejo de Edipo reprimido, esto sólo bastaría para hacerlo acreedor a contarse entre las conquistas más valiosas de la Humanidad"(10). Desde el punto de vista del funcionamiento cerebral, durante la etapa edípica ocurre un hecho de importancia capital: la mielinización interhemisférica comienza a ser suficiente para que se produzca un notable intercambio de información. Aunque esta mielinización interhemisférica es todavía incompleta a los 9 o 10 años de vida, durante el tercer año las relaciones 
interhemisféricas cambian drásticamente y el hemisferio izquierdo -el hemisferio del lenguaje- se vuelve dominąte respecto al hemisferio derecho. Por tanto, el comienzo de la etapa edípica, un periodo evolutivo crítico psicológica y neuroanatómicamente, coincidiría con un cambio radical en el procesamiento de la información. La maduración del SNC permitiría a los hemisferios cerebrales funcionar de una manera más coordinada y consolidaría el funcionamiento de los sistemas mnémicos relacionados con el logro de un self cohesivo. En opinión de Levin y otros autores (7), el éxito de la transición desde la etapa preedípica a la edípica dependería de la capacidad cerebral para coordinar diversas funciones interhemisféricas, entre ellas, la integración del funcionamiento según el proceso primario -hemisferio derecho- con el funcionamiento según el proceso secundario -hemisferio izquierdo. Otra consecuencia de la colaboración interhemisférica sería la puesta en marcha de nuevos y más maduros -neuróticos- mecanismos de defensa. De hecho, la represión no sería sino el resultado de cierto bloqueo del intercambio interhemisférico, que según Levin (7), un psicoanalista experto en neurofisiología y ciencia cognitiva, sería susceptible de verificarse mediante técnicas de neuroimagen. El conflicto psíquico sólo sería posible cuando las diferentes unidades funcionales cerebrales estuvieran conectadas pues, de lo contrario, esquemas o patrones arcaicos podrían coexistir aunque fueran mutuamente incompatibles. Sin embargo, el hallazgo potencialmente interesante para el investigador psicoanalítico sería, como hemos dicho, de otra naturaleza. ¿Qué sucedería si este proceso de mielinización interhemisférica presentara variaciones individuales significativas que, por causas constitucionales o ambientales, pudiera verse demorado o adelantado en el tiempo? La maduración fisiológica y psicológica puede llevar un ritmo diferente en un niño particular con respecto a la media, por ejemplo en la talla, sin que ello implique patología. Pero, desde un punto de vista psicodinámico, ¿daría lo mismo que un niño afrontara este periodo capital de su vida con una escasa mielinización interhemisférica? A priori, la respuesta es negativa. No parece que la dinámica pulsional edípica pueda ser experimentada y elaborada de manera idéntica. Un cerebro "bihemisférico" pondría a disposición del aparato psíquico una serie de mecanismos sublimatorios muy necesarios en el escenario edípico. En caso de demora en la mielinización, la colaboración interhemisférica precisaría que la información compartida siguiera transitando por estructuras arcaicas del SNC. De esta forma, el riesgo que cogniciones, afectos o conductas primitivas impregnaran los conflictos y relaciones objetales edípicas parecería evidente. La argumentacióńn que viene a continuación es especulativa, pero pretende dar un enfoque diferente a algunas de las mayores discrepancias que han tenido lugar en campo del psicoanálisis.

\section{El complejo de Edipo en el modelo kleiniano.}

Es de sobra conocido que la llamada escuela kleiniana ha defendido un comienzo mucho más precoz de la conflictiva pulsional edípica. También, que ha sido duramente criticada por proponer un escenario representacional integrado por fantasías inconscientes muy complejas cargado de afectos primitivos (11). Se ha reprochado que las representaciones mentales propuestas por Melanie Klein requerirían unas funciones 
cognitivas yoicas incompatibles con el desarrollo mental de niños tan pequeños (8). Sin embargo, la experiencia clínica muestra que el modelo kleiniano puede ser útil para comprender a algunos pacientes -aplicar la técnica kleiniana de manera ortodoxa a todos los pacientes no estaría justificado, en mi opinión, a la luz de los conocimientos neuropsicodinámicos actuales. Sin embargo, ¿qué pasaría si en un niño de 3-5 años la mielinización interhemisférica se retrasara? Las vicisitudes pulsionales serían las propias de la etapa edípica, su cerebro habría madurado y sería capaz de elaborar representaciones más abstractas, pero quizás el escenario representacional fuera diferente. El intercambio de información interhemisférico se vería obligado a discurrir por estructuras del SNC más arcaicas y el efecto del hemisferio del lenguaje, el izquierdo, sobre el derecho sería de eficacia limitada. Cabe preguntarse si el contenido de las fantasías inconscientes fruto de la rivalidad edípica no sufriese por este motivo severas distorsiones cognitivas y afectivas del tipo de las descritas por Melanie Klein. Por supuesto, la naturaleza de estas hipótesis es especulativa. Su importancia no radica tanto en la exactitud como en la propuesta: ¿podrían los hallazgos científicos corroborar o desestimar ciertas hipótesis psicodinámicas? En este sentido, ¿sería posible que el Edipo kleiniano estuviera presente en algunos pacientes tal y como fue descrito pero, la pretensión de adelantarlo en el tiempo, fuera fruto de un error? Dicho de otra manera, la observación clínica, el contenido de la fantasía inconsciente, habría sido correcta pero no su adelanto al primer año de vida. Por tanto, las representaciones con sus afectos primitivos no serían consecuencia de un escenario más precoz en el tiempo sino de una demora en la mielinización interhemisférica.

\section{El Complejo de Edipo en algunos trastornos graves de la personalidad.}

¿Cuál podría ser el resultado del mecanismo contrario: un adelanto de la mielinización interhemisférica? La mejora de la comunicación interhemisférica anticiparía la maduración cerebral. Ello posibilitaría ya al niño pequeño la elaboración de representaciones complejas de las relaciones triangulares con sus padres y, quizás, cierta activación pulsional edípica temprana. Sin embargo, este niño no habría tenido tiempo aún para elaborar las pulsiones orales o anales. Es decir, las fantasías edípicas precoces podrían estar muy cargadas de envidia u otros afectos primitivos propios de la edad biológica del niño. ¿Cómo podría afectar este adelanto madurativo al desarrollo del niño? Kernberg (12) ha propuesto un adelantamiento de las vicisitudes edípicas en ciertos pacientes con trastornos graves de la personalidad. De hecho, en contra de la opinión de otros notables psicoanalistas ha defendido que en el paciente borderline no se produce una regresión inespecífica, sino una regresión formal en el sentido de Freud: uso de una función de nivel inferior -escisión, por ejemplo- para el desarrollo actual de una persona, pero sin déficit global yoico. Es decir, el paciente borderline adulto se vería abocado a utilizar un mecanismo normal durante el desarrollo temprano -la escisión pasivapara poder mantener separadas las imágenes buenas/malas del self y del objeto -ahora, escisión activa, patológica- porque estarían muy cargadas de agresividad/envidia (13). ¿Es posible que el adelanto de la mielinización interhemisférica condicione un adelanto 
de ciertas vicisitudes edípicas, pero que la envidia/agresión temprana obligue a utilizar mecanismos de defensa muy drásticos con el fin de tolerar fantasías edípicas de gran contenido sadomasoquista? ¿Explicaría este proceso que, al menos en ciertos pacientes borderline, se produzca un desarrollo patológico y no, como proponen otros autores (14), una detención del desarrollo? No hay respuesta para estas preguntas todavía, pero lo realmente importante es que ciertas agrias polémicas entre psicoanalistas pueden dirimirse en el terreno de la investigación dejando a un lado querellas narcisistas personales (15).

\section{La dinámica consciente versus inconsciente.}

La teoría psicoanalítica sostiene que los procesos psíquicos son originalmente inconscientes y que algunos de ellos consiguen alcanzar la conciencia. $O$ dicho con terminología de la segunda tópica, el ello precede al yo, el cual se va diferenciando a partir del ello en virtud del contacto con la realidad exterior. Sin embargo, los estudios de observación de bebes realizados por Stern (8) indican que existe una dialéctica simultánea entre el ello y el yo, los cuales operarían por igual desde el inicio de la vida. Este sería un ejemplo de divergencia con la teoría clásica. Podría argumentarse que la observación de bebes no es comparable al encuadre analítico y por tanto sus hallazgos no serían aplicables a la teoría psicodinámica. En mi opinión, cabe otra postura, ¿existen más datos que puedan facilitar la comprensión del problema que nos ocupa? Por ahora no están disponibles, aunque un reciente descubrimiento (16) podría facilitar enormemente nuestro conocimiento actual de la consciencia. Un grupo de investigadores ha descubierto un grupo de neuronas corticales a las que ha llamado neuronas en espejo. Estas neuronas podrían tener la capacidad de "leer" las intenciones de los otros, fenómeno que para muchos autores podría estar en el origen de la consciencia (1).

En resumen, la búsqueda exclusiva de las bases neuroquímicas de las manifestaciones psicológicas complejas puede resultar muy empobrecedora. Sin embargo, la investigación propiamente psicoanalítica no tiene porque estar reñida con la revisión de las hipótesis científicas que subyacen a la teoría. Son ya muchos los autores $(7-8,15,17)$ que opinan que esta tarea es posible y la única limitación admisible es la magnitud del conocimiento científico actual. 


\section{DEBATES}

\section{BIBLIOGRAFIA}

(1) Paniagua, C., "Consciencia, Cientificismo y Multideterminismo en Psicoanálisis", Rev. Asoc. Esp. Neuropsiq., 2000, 20, 76, pp. 67-78

(2) Wyss, D., Las escuelas de psicología profunda, Madrid, Gredos, 1961.

(3) Miller, L., "Brain and self: Toward a neuropsychodynamic model of ego autonomy and personality", J. American Academy Psychoanalysis, 1991,19, 2, pp. 213-234.

(4) Shevrin, H., " The fate of the five metapsychological principles", Psychoanalytic Inquiry, 1948, 4, pp. 33-58.

(5) Chugani, H.T.; Phelps, M.E., "Maturational changes in cerebral function in infants determined by FDG positron emission tomography", Science, 1986, 231, pp. 840-843.

(6) Gao, J.H., "Cerebellum implicated in sensory acquisition and discriminating rather than motor control”, Science,1996, 272, pp. 545-547.

(7) Levin, F.M., Mapping de mind, New York, Analytic Press, 1991.

(8) Stern, D.N., The interpersonal world of the infant, New York, Basic Books, 1985.

(9) Kim, S.; Ugurbil, K.; Strick, P.L., “Activation of a cerebellar output nucleus during congnitive processing”, Science, 1994, 265, pp. 949-951.

(10) Freud. "Introducción al narcisismo", 1914, O.C., Madrid, Biblioteca Nueva, 1972.

(11) Ogden, T.H., La matriz de la mente, Madrid, Tecnipublicaciones, 1989.

(12) Kernberg, O.F. Desórdenes fronterizos y narcisismo patológico, Buenos Aires, Paidos, 1979.

(13) Lana, F., "El enfermo borderline: etiología y patogenia", Rev. Psicoanálisis y Psicoterapia, 1996, 3, pp.81-93.

(14) Masterson, J.F., "Diagnóstico y tratamiento del síndrome borderline en adolescentes", Confrontaciones Psiquiátricas, 1983.

(15) Lana, F.; Rodado, J.; Santamaría C., “Aproximación a los fundamentos neuropsicodinámicos de la teoría psicoanalítica”, Rev. Psicoanálisis y Psicoterapia, 1997, 4, pp. 25-59.

(16) Gallesse, V.; Goldman, A., "Mirror neurons and the simulation theory of mind-reading", Trends in Cognitive Sciences, 1998, 2, pp. 493-497.

(17) Gedo, J.E., "Foreword, en Levin, F.M. (ed.), Mapping the mind, New York, The Analytic Press, 1991.

(*) Psiquiatra. Psicoanalista.

Miembro del Centro Psicoanalítico de Madrid y de la International Federation of Psychoanalytic Societies.

Jefe del Departamento de Salud Mental

Centres Assistencials Torribera

Santa Coloma de Gramenet (Barcelona).

\section{CORRESPONDENCIA:}

Dr. Fernando Lana Moliner

Departament de Salut Mental

Centres Assistencials Torribera

C/ Prat de la Riba, 171 - 08921 Santa Coloma de Gramenet (Barcelona). 